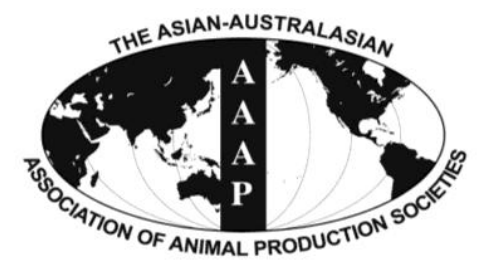

Asian Australas. J. Anim. Sci.

Vol. 26, No. 10 : 1446-1458 October 2013

http://dx.doi.org/10.5713/ajas.2013.13066

www.ajas.info

pISSN 1011-2367 elSSN 1976-5517

\title{
Influence of Dietary Supplementation of Condensed Tannins through Leaf Meal Mixture on Intake, Nutrient Utilization and Performance of Haemonchus contortus Infected Sheep
}

\author{
A. K. Pathak*, Narayan Dutta, P. S. Banerjee, A. K. Pattanaik, and K. Sharma ${ }^{1}$ \\ Centre of Advanced Faculty Training in Animal Nutrition, Indian Veterinary Research Institute, \\ Izatnagar-243 122 (U.P.), India
}

\begin{abstract}
The study assessed the effect of dietary supplementation of leaf meal mixture (LMM) containing condensed tannins (CT) on feed intake, nutrient utilization and performance of sheep infected with Haemonchus contortus. Eighteen adult sheep of similar age and body weight $(25.03 \pm 1.52)$ were included in this study and out of these, 12 sheep were infected with single dose of infective third stage larvae of $H$. contortus at 2,000 larvae per sheep. The experimental sheep were allocated in three different groups' i.e. negative control (NC; no infection), control $(\mathrm{C} ; H$. contortus infected) and treatment $(\mathrm{T} ; H$. contortus infected $+\mathrm{CT}$ at $1.5 \%$ of the DM through LMM) and the experiment was conducted for a period of $90 \mathrm{~d}$. The intake of dry matter (DM), organic matter (OM) and digestibility of DM, OM, neutral detergent fibre (NDF) and acid detergent fibre (ADF) were comparable among three animal groups. However, digestibility of crude protein $(\mathrm{CP})$ and ether extract $(\mathrm{EE})$ were significantly $(\mathrm{p}<0.05)$ higher in NC group as compared to both $\mathrm{C}$ and $\mathrm{T}$ groups. Nitrogen $(\mathrm{N})$ retention $(\mathrm{g} / \mathrm{d}$ or $\%$ of $\mathrm{N}$ intake) was significantly $(\mathrm{p}=0.038)$ lower in $\mathrm{C}$ group as compared to $\mathrm{T}$ and $\mathrm{NC}$ groups. Daily intake $\left(\mathrm{g} / \mathrm{kg} \mathrm{W}^{0.75}\right)$ of digestible crude protein (DCP), digestible organic matter (DOM) and total digestible nutrient (TDN) did not differ significantly $(\mathrm{p}<0.05)$ in the three groups. Haemoglobin $(\mathrm{Hb})$ and packed cell volume $(\mathrm{PCV})$ were significantly $(\mathrm{p}<0.001)$ higher in treatment group as compared to control. The level of $\mathrm{Hb}$ and PCV reduced $(\mathrm{p}<0.001)$ after 30 days of experimental feeding. CT significantly $(p<0.001)$ reduced serum urea in T group as compared to NC and C groups. Serum proteins differed significantly ( $<<0.01$ ) among the three groups. The activity of serum enzymes AST, ALT, ALP and LDH were also statistically non significant $(p<0.05)$ among treatments. The weight of abomasal lymph nodes (ALN) in T group was higher $(\mathrm{p}<0.05)$ than in $\mathrm{C}$ group. Treatment group had lower $(\mathrm{p}<0.05)$ total worms and fecal egg count compared to control group. It may be concluded that dietary supplementation of CT through LMM significantly improved the $\mathrm{N}$ retention, and inhibited the different developmental stages of Haemonchus contortus in experimental sheep. (Key Words: Condensed Tannins, Haemonchus contortus, Leaf Meal Mixture, Nutrient Utilization, Performance, Sheep)
\end{abstract}

\section{INTRODUCTION}

Ruminant production in the tropics is entirely dependent on crop residue based feeds. Cereal crop residues are usually deficient in nitrogen, minerals and vitamins and high in lignin, and do not provide sufficient nutrients to meet the requirements of the animals, when fed alone (Leng, 1990). The poorly nourished animals are more susceptible to high prevalence and frequent outbreaks of infectious diseases including gastrointestinal nematodes (GIN) leading

\footnotetext{
* Corresponding Author: A. K. Pathak. Faculty of Veterinary Science and Animal Husbandry, SKUAST-Jammu, India. Tel: +91-9419774635, Fax: +91-1923-250920, E-mail: drakp_74 @ rediffmail.com

${ }^{1}$ ICAR, New Delhi, India.

Submitted Jan. 24, 2013; Accepted Apr. 8, 2013; Revised May 17, 2013
}

to further adverse effects on the productivity of animals (Pathak and Pal, 2008; Pathak, 2011). In the event of inadequate nutrient availability, many bodily functions are penalized, including immune functions, which are important determinants in regulating the establishment and survival of GINs within the animal. However, dietary supplementation is known to increase host's resistance to GIN and thereby may contribute to effective augmentation in the animals' productivity.

Haemonchus contortus is one of the most pathogenic and widely distributed blood sucking abomasal nematodes of sheep and goats. Adult $H$. contortus can suck $0.05 \mathrm{~mL}$ of blood/ worm/ day (Rowe et al., 1988). Sub-clinical GIN infections occasionally depress feed intake, utilization and production (mortality and weight loss), and can impair tissue deposition and skeletal growth (Parkins and Holmes, 
1989). The customary mode of control of these GINs has been based on the repeated use of chemical anthelmintics. However, drug resistance has become an important issue in small ruminant husbandry, especially when anthelmintics are applied at high levels and increasing frequency and inappropriate doses (Pandey et al., 2001). Moreover, growing interest in global organic food production in recent years primarily due to adverse impact of intensive farming on environment, animal health and as well as consumers concern for food safety restricts use of chemicals in animal feeds (IFOM, 2006). Thus, alternative environment friendly sustainable novel strategies are required, which could reduce the exclusive reliance on anthelmintic treatment.

Use of phyto-chemicals like condensed tannins (CT) is becoming preferable and may offer better control than anthelmintics to treat GIN (Chandrawathani et al., 2000; Chaichisemsari et al., 2011; Hassanpour et al., 2011). The CT could complex with nutrients and inhibits nutrient availability for larval growth or decrease GIN infection metabolism directly through inhibition of oxidative phosphorylation (Scalbert, 1991), causing larval death (Athanasiadou et al., 2001). This raises the possibility that feeding locally available tropical tree leaves containing CT may be an alternative method for controlling $H$. contortus infections, especially in tropics and subtropics. Therefore, potential source and optimum level of CT to be used in the diets to reduce $H$. contortus load in sheep warrants investigation. This may provide some advantage to conquer the limitations of ruminants by reducing $H$. contortus parasites with the strategic use of tanniferous tree leaves, which otherwise remain under-utilized as animal feed. Keeping this in view, the present experiment investigated the effect of CT containing LMM on nutrient utilization, balances, biochemical constituents and control of $H$. contortus in infected sheep.

\section{MATERIALS AND METHODS}

\section{Experimental animals and feeding}

Eighteen adult sheep of either sex, similar age and body weight $(25.03 \pm 1.52)$ were selected from the well maintained herd for experimental study. Out of 18 sheep, 12 sheep were infected with single dose of infective third stage larvae of $H$. contortus at 2,000 larvae per sheep. All the sheep were allocated in three different groups i.e. Negative control (NC; no infection), control (C; H. contortus infected) and treatment $(\mathrm{T} ; H$. contortus infected+CT at $1.5 \%$ of the DM through LMM). A negative control group was taken to compare their performance with infected control and treatment groups. All sheep were housed individually in a well-ventilated shed, with facilities for individual feeding. All sheep were offered a basal diet of wheat straw ad libitum along with required amount of concentrate mixture for a period of 90 days to meet their nutrient requirements for maintenance as per Kearl (1982). One hundred grams of oat hay was given to each sheep per day to meet their vitamin-a requirement. Dried LMM of Ficus infectoria and Psidium guajava (in the ratio of 70:30) was incorporated in the diet of treatment group by replacing concentrate mixture so as to bring the diet iso-nitrogenous (22\% CP in concentrate mixture) and CT content to 1.5 percent of the dry matter. The feeding of CT was started from the same day of oral dosing of $H$. contortus larvae in $\mathrm{T}$ group. A metabolism trial for $6 \mathrm{~d}$ collection period was conducted towards the end of feeding trial to assess the nutrient intake, digestibility and balances of nitrogen $(\mathrm{N})$, calcium $(\mathrm{Ca})$ and inorganic phosphorus $(\mathrm{P})$. The collection of faecal and urine samples was made after 3 days adaptation of the sheep in metabolic cages. The records of daily faeces and urine voided $(24 \mathrm{~h})$ were maintained during 6 days collection. Blood samples were collected through jugular vein puncture at $0,30,60$ and 90 days of experimentation, serum was separated and preserved at $-20^{\circ} \mathrm{C}$ until analysed. Another $2 \mathrm{~mL}$ blood sample was collected in tubes containing ethylene diamine tetra acetate (1 mg/mL blood), for haematological studies.

\section{Chemical analysis}

The CT content of LMM was estimated by Butanol-HCl method (Makkar, 2000). Representative samples of feed offered, residue left, faeces voided and urine excreted were processed and analyzed for proximate principles (AOAC, 2000). The Ca content in the feed, residue, faeces and urine was determined by the precipitation method (Talpatra et al., 1940), however, $P$ content of the above samples was determined spectrophotometrically adopting the metavandate method (AOAC, 2000). NDF and ADF content of feeds, residue and faeces samples were estimated by the method of Van Soest et al. (1991). NDF was assayed with sodium sulphite, in the NDF reagent without alpha-amylase and the results were expressed with residual ash. Gross energy of feed samples was determined by adiabatic bomb calorimeter (Toshniwal Microprocessor Bomb Calorimeter; cat. No. Cc. 01/m3) along with computer operated software which gives the gross energy value of feed samples in (cal/g) unit. All the biochemical parameters were estimated by using diagnostic kits (DIA Lab GmbH, Austria) marketed by Diagnova, a Division of RFCL limited, New Delhi, India. Haemoglobin $(\mathrm{Hb})$ and packed cell volume (PCV) were estimated in whole blood immediately after blood collection by cyanomethaemoglobin method (Dacie and Lewis, 1969) and Wintrobe's tube, respectively. The total eosinophils were measured as the method described by Sastry (1989). Serum glucose concentration was determined colorimetrically (Barham and Trinder, 1972). The serum protein and albumin content were measured as per Vatzidis 
(1977) and Doumas et al. (1971), respectively. Serum urea was estimated by the method of Hallett and Cook (1971). Serum cholesterol and creatinine were measured as per Richmond (1973) and (Newman and Price, 1999), respectively. Activity of serum enzymes, alanine aminotransferase (ALT) and aspartate aminotransferase (AST) were determined as per standard methods described by Thefeld et al. (1974) whereas alkaline phosphatase (ALP) and lactate dehydrogenase (LDH) were determined as per Bretaudiere et al. (1977) and Wroblewski and Duean (1955), respectively.

\section{Animal slaughter and sampling}

At the end of feeding trial $(90 \mathrm{~d})$, the infected sheep of both groups $(\mathrm{C}$ and $\mathrm{T})$ were slaughtered as per standard protocol. The sheep were kept off feed over night $(16 \mathrm{~h})$ with free access to drinking water. They were weighed immediately prior to slaughter and recorded it as preslaughter weight (PSW) of sheep. The sheep were slaughtered by adopting standard procedure. After slaughter, the sheep bodies were hung on the rail to remove the skin, head and feet and weight was recorded as dressed weight. The abdominal cavity was opened. The various parameters were studied in slaughtered sheep viz. carcass weight, weights of gastrointestinal tract (GIT), pre-scapular (PSLN) and abomasal lymph nodes (ALN), dressing percentage, and total worm counts. The abomasums of slaughtered sheep was removed and opened. The contents were emptied and washed with water. Worms were removed from abomasal tissue and fold using fine forceps and then counted total worms. Faecal egg counts (FECs) were made using the modified McMaster technique (Anonymous, 1984). The hatching percentage/larval development from faecal samples of both infected groups were made by faecal culture technique as described by Urquhart et al. (1996). The infective 3rd stage larvae were counted as per standard protocol.

\section{Statistical analysis}

The results obtained were subjected to analysis of variance using SPSS 11.0 software and treatment means were ranked using Duncan's multiple range tests. The periodic alterations in biochemical parameters were analyzed using repeated measures design (General linear model, Multivariate). Significance of treatments with respect to different characters was declared at $\mathrm{p}<0.05$ unless otherwise stated. All the statistical procedure was done as per Snedecor and Cochran (1994).

\section{RESULTS}

\section{Chemical composition of feeds}

The chemical composition (\% DM basis) of concentrate mixture, leaf meal mixture, oat hay and wheat straw offered to sheep for a period of 90 days and during metabolism trial is presented in the Table 1.

\section{Intake and digestibility of nutrients}

The daily nutrient intake, digestibility and their utilization by sheep during the metabolism trial under three different groups ( $\mathrm{NC}, \mathrm{C}$, and $\mathrm{T}$ ) is given in the Table 2 . Daily intake of DM and OM was statistically similar among the three groups (NC, C, and T). Similarly, roughage intake did not differ significantly among dietary treatments. While, concentrate intake was significantly $(\mathrm{p}<0.01)$ lower in $\mathrm{T}$ group as compared to $\mathrm{NC}$ and $\mathrm{C}$. The digestibility coefficients (\%) of $\mathrm{CP}$ and $\mathrm{EE}$ were significantly higher $(\mathrm{p}<0.017$ and $\mathrm{p}<0.001)$ in $\mathrm{NC}$ as compared to both $\mathrm{C}$ and $\mathrm{T}$ groups, however, digestibility of DM, OM, NDF, and ADF did not differ significantly irrespective of treatments.

\section{Nutritive value, plane of nutrition and nutrient balance}

The daily intake $\left(\mathrm{g} / \mathrm{kg} \mathrm{W}^{0.75}\right)$ of DCP, DOM and TDN was comparable irrespective of treatment groups (Table 2). Similarly, N intake and excretion (g/d) through faeces did not differ significantly among three groups, however, urinary- $\mathrm{N}$ excretion $(\mathrm{g} / \mathrm{d})$ and percent of $\mathrm{N}$ intake was significantly $(\mathrm{p}<0.001)$ lower in $\mathrm{T}$ as compared to $\mathrm{C}$ group. Similarly, N-retention $(\mathrm{g} / \mathrm{d}$; as $\%$ of intake or as \% of absorbed $\mathrm{N}$ ) was significantly $(\mathrm{p}<0.038)$ lower in $\mathrm{C}$ as

Table 1. Chemical composition of feeds (\% DM)

\begin{tabular}{lccrr}
\hline Attributes & Wheat straw & Oat hay & Concentrate mixture & Leaf meal mixture \\
\hline Organic matter & 92.72 & 90.95 & 91.79 & 90.82 \\
Crude protein & 3.43 & 9.10 & 22.10 & 10.80 \\
Ether extract & 1.55 & 1.63 & 2.98 & 3.21 \\
Total Ash & 7.28 & 9.05 & 8.21 & 9.18 \\
Acid insoluble ash & 5.15 & 3.68 & 1.20 & 2.98 \\
Calcium & 0.2 & 0.34 & 1.24 & 1.61 \\
Phosphorus & 0.04 & 0.33 & 0.73 & 0.20 \\
Neutral detergent fibre & 84.75 & 70.17 & 35.15 & 57.29 \\
Acid detergent fibre & 55.68 & 46.20 & 11.03 & 44.97 \\
Gross energy (kcal/kg DM) & $3,989.74$ & $4,013.10$ & $4,233.15$ & $4,121.16$ \\
Condensed tannins & - & - & - & 10.44 \\
\hline
\end{tabular}


Table 2. Effect of CT supplementation on nutrients intake and digestibility by sheep

\begin{tabular}{|c|c|c|c|c|c|}
\hline \multirow{2}{*}{ Attributes } & \multicolumn{3}{|c|}{ Group* } & \multirow{2}{*}{ SEM } & \multirow{2}{*}{$\mathrm{p}$ values } \\
\hline & $\mathrm{NC}$ & $\mathrm{C}$ & $\mathrm{T}$ & & \\
\hline \multicolumn{6}{|c|}{ Metabolic body size } \\
\hline $\mathrm{kg} \mathrm{W}^{0.75}$ & 11.70 & 11.44 & 11.57 & 0.35 & 0.96 \\
\hline \multicolumn{6}{|c|}{ Nutrient intake $\left(\mathrm{g} / \mathrm{kg} \mathrm{W}^{0.75}\right)$} \\
\hline $\mathrm{DM}$ & 52.50 & 58.05 & 59.11 & 3.69 & 0.758 \\
\hline $\mathrm{OM}$ & 50.42 & 55.75 & 56.11 & 3.40 & 0.770 \\
\hline Concentrate & $16.09^{\mathrm{b}}$ & $16.55^{\mathrm{b}}$ & $12.47^{\mathrm{a}}$ & 0.55 & 0.000 \\
\hline LMM & 0.00 & 0.00 & 9.93 & 1.23 & 0.000 \\
\hline Roughage & 36.41 & 41.50 & 36.71 & 3.20 & 0.789 \\
\hline DCP & 3.36 & 3.19 & 3.34 & 0.13 & 0.859 \\
\hline DOM & 29.8 & 31.77 & 32.63 & 1.86 & 0.842 \\
\hline TDN & 31.36 & 33.36 & 34.26 & 1.95 & 0.842 \\
\hline \multicolumn{6}{|c|}{ Nutrient digestibility (\%) } \\
\hline $\mathrm{DM}$ & 52.34 & 49.11 & 51.33 & 0.96 & 0.394 \\
\hline $\mathrm{OM}$ & 60.42 & 57.16 & 57.91 & 0.96 & 0.369 \\
\hline $\mathrm{CP}$ & $64.41^{\mathrm{b}}$ & $58.03^{\mathrm{a}}$ & $59.74^{\mathrm{a}}$ & 1.01 & 0.017 \\
\hline $\mathrm{EE}$ & $49.82^{\mathrm{b}}$ & $40.63^{\mathrm{a}}$ & $44.37^{\mathrm{a}}$ & 1.20 & 0.001 \\
\hline $\mathrm{NDF}$ & 49.92 & 46.53 & 47.86 & 0.90 & 0.317 \\
\hline $\mathrm{ADF}$ & 39.95 & 36.94 & 38.37 & 1.17 & 0.605 \\
\hline \multicolumn{6}{|c|}{ Nutrient density (\%) } \\
\hline DCP & 6.80 & 5.64 & 5.76 & 0.28 & 0.174 \\
\hline TDN & 61.32 & 57.83 & 57.76 & 1.13 & 0.361 \\
\hline \multicolumn{6}{|l|}{ CT intake } \\
\hline$\%$ of DM diet & 0.00 & 0.00 & 1.72 & 0.20 & 0.000 \\
\hline
\end{tabular}

${ }^{\mathrm{ab}}$ Means with different superscripts within a row differ significantly. * $\mathrm{NC}=$ Negative control; $\mathrm{C}=$ Control; $\mathrm{T}=$ Treatment.

$\mathrm{DM}=$ Dry matter, $\mathrm{OM}=$ Organic matter, $\mathrm{LMM}=$ Leaf meal mixture, $\mathrm{DCP}=$ Digestible crude protein, $\mathrm{DOM}=$ Digestible organic matter, $\mathrm{TDN}=$ Total digestible nutrients, $\mathrm{CP}=$ Crude protein, $\mathrm{EE}=$ Ether extract, $\mathrm{NDF}=$ Neutral detergent fibre, $\mathrm{ADF}=$ Acid detergent fibre, $\mathrm{CT}=\mathrm{Condensed}$ tannins .

compared to T group, while NC was comparable to T group (Table 3). The $\mathrm{Ca}$ and $\mathrm{P}$ intakes, out go and their retention (g/d) did not differ significantly among groups.

\section{Blood-biochemical profile}

Haematological parameters: $\mathrm{Hb}$ and $\mathrm{PCV}$ were significantly $(\mathrm{p}<0.001)$ higher in treatment group as compared to control, however, NC had higher $(\mathrm{p}<0.001)$ values than treatment group (Table 4). The level of $\mathrm{Hb}$ and PCV reduced $(\mathrm{p}<0.001)$ after 30 days of experimental feeding. However, eosinophil counts $(\%)$ were significantly $(\mathrm{p}<0.001)$ higher in treatment group followed by $\mathrm{C}$ and $\mathrm{NC}$. The period and treatment interaction was also significant $(\mathrm{p}<0.001)$.

Biochemical parameters: Various biochemical constituents'viz. serum glucose, calcium, phosphorus cholesterol, creatinine levels, serum enzymes (ALP, ALT, AST and LDH), serum proteins and the serum urea levels are presented in the Table 5, 6, and 7. The level of serum glucose, calcium, inorganic phosphorus, cholesterol and creatinine contents were similar among three groups. No significant difference was evident in serum enzymes irrespective of treatments. However, AST activity were significantly $(\mathrm{p}<0.001)$ increased in all the experimental animals at 90 days of examination. The CT supplementation significantly $(\mathrm{p}<0.001)$ reduced serum urea level in $\mathrm{T}$ group as compared to NC and C groups. The total protein (TP), albumin and globulin among three different groups differed significantly $(\mathrm{p}<0.01)$. The TP and globulin were significantly $(\mathrm{p}<0.001)$ higher in $\mathrm{T}$ group as compared to $\mathrm{C}$ group. The NC group was also comparable to $\mathrm{T}$ group. The albumin level was also found to be statistically lower $(\mathrm{p}<0.01)$ under $\mathrm{T}$ group as compared to $\mathrm{C}$ group. The level of albumin decreased from 30 days experimental period and onwards.

\section{Slaughtered parameters and $\boldsymbol{H}$. contortus worm burden}

Pre slaughter weight, carcass weight and weight of GIT did not differ significantly between both $\mathrm{C}$ and $\mathrm{T}$ groups (Table 8). Significantly higher dressing percentage was evident in $\mathrm{T}$ group as compared to $\mathrm{C}$ group. The pooled weight of pre-scapular lymph nodes was comparable between both $\mathrm{C}$ and $\mathrm{T}$ groups; however, abomasal lymph nodes weight was significantly higher $(\mathrm{p}<0.05)$ in $\mathrm{T}$ group. Total worms and FEC and were found to be higher $(\mathrm{p}<0.05)$ in the abomasum of $\mathrm{C}$ group as compared to $\mathrm{T}$ group. Similarly, significantly higher hatching percentage/ developed larvae $\left(\mathrm{L}_{3}\right)$ was observed in $\mathrm{C}$ group as compared 
Table 3. Effect of condensed tannins supplementation on nutrient balance of sheep

\begin{tabular}{|c|c|c|c|c|c|}
\hline \multirow{2}{*}{ Attributes } & \multicolumn{3}{|c|}{ Group* } & \multirow{2}{*}{ SEM } & \multirow{2}{*}{$\mathrm{p}$ value } \\
\hline & $\mathrm{NC}$ & $\mathrm{C}$ & $\mathrm{T}$ & & \\
\hline \multicolumn{6}{|l|}{ Nitrogen balance $(\mathrm{g} / \mathrm{d})$} \\
\hline $\mathrm{N}$ intake & 9.84 & 10.01 & 10.25 & 0.45 & 0.938 \\
\hline Faecal- N & 3.52 & 4.13 & 4.28 & 0.18 & 0.186 \\
\hline Urinary-N & $3.33^{\mathrm{b}}$ & $4.08^{\mathrm{c}}$ & $2.72^{\mathrm{a}}$ & 0.17 & 0.001 \\
\hline Total-N excretion & $6.85^{\mathrm{a}}$ & $8.21^{\mathrm{b}}$ & $7.00^{\mathrm{a}}$ & 0.24 & 0.032 \\
\hline $\mathrm{N}$ retained $(\mathrm{g} / \mathrm{d})$ & 2.99 & 1.80 & 3.25 & 0.34 & 0.183 \\
\hline $\mathrm{N}$ retained $\%$ of intake & $29.72^{\mathrm{b}}$ & $15.38^{\mathrm{a}}$ & $30.73^{\mathrm{b}}$ & 2.86 & 0.038 \\
\hline $\mathrm{N}$ retained $\%$ of absorbed & $46.33^{\mathrm{ab}}$ & $25.37^{\mathrm{a}}$ & $52.74^{\mathrm{c}}$ & 4.74 & 0.036 \\
\hline \multicolumn{6}{|l|}{ Calcium balance (g/d) } \\
\hline Ca intake & 3.42 & 3.44 & 4.55 & 0.23 & 0.069 \\
\hline Faecal-Ca & 1.51 & 1.67 & 2.19 & 0.13 & 0.083 \\
\hline Urinary-Ca & 0.19 & 0.01 & 0.01 & 0.02 & 0.112 \\
\hline Ca- retained $(\mathrm{g} / \mathrm{d})$ & 1.73 & 1.68 & 2.26 & 0.12 & 0.088 \\
\hline Ca-retained $\%$ of $\mathrm{Ca}$ intake & 51.41 & 49.28 & 49.49 & 1.69 & 0.866 \\
\hline \multicolumn{6}{|l|}{ Phosphorus balance (g/d) } \\
\hline$P$ intake & 1.76 & 1.77 & 1.71 & 0.06 & 0.925 \\
\hline Faecal-P & 0.93 & 1.01 & 0.91 & 0.04 & 0.625 \\
\hline Urinary-P & 0.02 & 0.02 & 0.02 & 0.002 & 0.775 \\
\hline P- retained $(\mathrm{g} / \mathrm{d})$ & 0.81 & 0.75 & 0.79 & 0.03 & 0.784 \\
\hline P-retained $\%$ of $\mathrm{Ca}$ intake & 46.15 & 42.50 & 46.11 & 1.05 & 0.278 \\
\hline
\end{tabular}

${ }^{\text {abc }}$ Means with different superscripts within a row differ significantly.

$* \mathrm{NC}=$ Negative control; $\mathrm{C}=\mathrm{Control} ; \mathrm{T}=$ Treatment. $\mathrm{N}=$ Nitrogen, $\mathrm{Ca}=$ Calcium, $\mathrm{P}=$ Phosphorus .

to T group (Table 9).

\section{DISCUSSION}

\section{Chemical composition of feed}

The chemical composition of concentrate, LMM, oat hay and wheat straw used in the experiment was comparable with the values reported by many workers (Patra et al., 2006; Dey et al., 2008). The NDF and ADF values were higher in LMM as compared to concentrate. This could be attributed to the high cell-wall constituents usually present in leaf meal (Anbarasu et al., 2004; Dey et

Table 4. Effect of condensed tannins on haematological profiles in H. contortus infected sheep

\begin{tabular}{|c|c|c|c|c|c|c|c|c|}
\hline \multirow{2}{*}{ Attributes } & \multicolumn{4}{|c|}{ Periods* } & \multirow{2}{*}{$\mathrm{GM} \pm \mathrm{SE}$} & \multicolumn{3}{|c|}{$\mathrm{p}$ values } \\
\hline & $0 \mathrm{~d}$ & $30 \mathrm{~d}$ & $60 \mathrm{~d}$ & $90 \mathrm{~d}$ & & $\mathrm{G}$ & $\mathrm{P}$ & $\mathrm{G} \times \mathrm{P}$ \\
\hline \multicolumn{9}{|c|}{ Haemoglobin (g/dL) } \\
\hline $\mathrm{NC}$ & 10.17 & 10.85 & 10.46 & 10.29 & $10.44^{\mathrm{c}} \pm 0.19$ & 0.000 & 0.000 & 0.002 \\
\hline $\mathrm{C}$ & 10.22 & 8.57 & 8.15 & 6.84 & $8.44^{\mathrm{a}} \pm 0.33$ & & & \\
\hline $\mathrm{T}$ & 9.94 & 9.16 & 9.00 & 8.89 & $9.25^{\mathrm{b}} \pm 0.16$ & & & \\
\hline $\mathrm{PM} \pm \mathrm{SE}$ & $10.11^{\mathrm{c}} \pm 0.18$ & $9.53^{\mathrm{bc}} \pm 0.32$ & $9.20^{\mathrm{ab}} \pm 0.30$ & $8.67^{\mathrm{a}} \pm 0.41$ & & & & \\
\hline \multicolumn{9}{|l|}{ PCV \% } \\
\hline $\mathrm{NC}$ & 40.50 & 42.33 & 41.67 & 41.17 & $41.42^{\mathrm{c}} \pm 0.73$ & 0.000 & 0.000 & 0.001 \\
\hline $\mathrm{C}$ & 40.67 & 31.83 & 29.67 & 21.67 & $30.96^{\mathrm{a}} \pm 1.78$ & & & \\
\hline $\mathrm{T}$ & 39.83 & 36.17 & 35.67 & 34.17 & $36.46^{\mathrm{b}} \pm 1.04$ & & & \\
\hline $\mathrm{PM} \pm \mathrm{SE}$ & $40.33^{\mathrm{c}} \pm 1.23$ & $36.78^{\mathrm{b}} \pm 1.42$ & $35.67^{\mathrm{b}} \pm 1.59$ & $32.33^{\mathrm{a}} \pm 2.13$ & & & & \\
\hline \multicolumn{9}{|c|}{ Eosinophil counts (\%) } \\
\hline $\mathrm{NC}$ & 2.67 & 2.33 & 2.83 & 1.83 & $2.42^{\mathrm{a}} \pm 0.16$ & 0.000 & 0.000 & 0.000 \\
\hline $\mathrm{C}$ & 2.33 & 7.17 & 5.50 & 4.33 & $4.83^{\mathrm{b}} \pm 0.43$ & & & \\
\hline $\mathrm{T}$ & 2.50 & 11.83 & 9.17 & 7.50 & $7.75^{c} \pm 0.75$ & & & \\
\hline $\mathrm{PM} \pm \mathrm{SE}$ & $2.50^{\mathrm{a}} \pm 0.17$ & $7.11^{\mathrm{d}} \pm 0.98$ & $5.83^{c} \pm 0.68$ & $4.55^{\mathrm{b}} \pm 0.62$ & & & & \\
\hline
\end{tabular}

abcd Means with different superscripts within a row and column differ significantly. $*$ NC $=$ Negative control; $\mathrm{C}=$ Control; $\mathrm{T}=$ Treatment; $\mathrm{d}=$ Days. $\mathrm{PM}=$ Pooled mean, $\mathrm{GM}=$ Group mean, $\mathrm{PCV}=$ Packed cell volume . 
Table 5. Effect of condensed tannins on various biochemical profiles in $H$. contortus infected sheep

\begin{tabular}{|c|c|c|c|c|c|c|c|c|}
\hline \multirow{2}{*}{ Attributes } & \multicolumn{4}{|c|}{ Periods* } & \multirow{2}{*}{$\mathrm{GM} \pm \mathrm{SE}$} & \multicolumn{3}{|c|}{$\mathrm{p}$ values } \\
\hline & $0 \mathrm{~d}$ & $30 \mathrm{~d}$ & $60 \mathrm{~d}$ & $90 \mathrm{~d}$ & & $\mathrm{G}$ & $\mathrm{P}$ & $\mathrm{G} \times \mathrm{P}$ \\
\hline \multicolumn{9}{|c|}{ Serum glucose (mg/dL) } \\
\hline $\mathrm{NC}$ & 39.46 & 42.94 & 44.45 & 46.34 & $43.30 \pm 1.24$ & 0.139 & 0.027 & 0.949 \\
\hline $\mathrm{C}$ & 38.83 & 43.78 & 43.11 & 42.46 & $42.04 \pm 1.04$ & & & \\
\hline $\mathrm{T}$ & 42.22 & 46.30 & 45.92 & 45.70 & $45.04 \pm 0.90$ & & & \\
\hline $\mathrm{PM} \pm \mathrm{SE}$ & $40.17^{\mathrm{a}} \pm 1.45$ & $44.34^{\mathrm{b}} \pm 1.15$ & $44.50^{\mathrm{b}} \pm 1.16$ & $43.83^{\mathrm{b}} \pm 1.32$ & & & & \\
\hline \multicolumn{9}{|c|}{ Serum calcium (mg/dL) } \\
\hline $\mathrm{NC}$ & 10.04 & 10.17 & 9.99 & 10.01 & $10.05 \pm 0.23$ & 0.197 & 0.127 & 0.649 \\
\hline $\mathrm{C}$ & 10.37 & 9.84 & 8.89 & 8.72 & $9.46 \pm 0.28$ & & & \\
\hline $\mathrm{T}$ & 10.23 & 10.01 & 9.92 & 9.36 & $9.88 \pm 0.20$ & & & \\
\hline $\mathrm{PM} \pm \mathrm{SE}$ & $10.21^{\mathrm{b}} \pm 0.32$ & $10.01^{\mathrm{ab}} \pm 0.23$ & $9.60^{\mathrm{ab}} \pm 0.23$ & $9.36^{\mathrm{a}} \pm 0.30$ & & & & \\
\hline \multicolumn{9}{|c|}{ Serum phosphorus (mg/dL) } \\
\hline $\mathrm{NC}$ & 5.89 & 6.03 & 5.75 & 5.66 & $5.83 \pm 0.12$ & 0.621 & 0.106 & 0.933 \\
\hline $\mathrm{C}$ & 5.91 & 5.73 & 5.52 & 5.47 & $5.66 \pm 0.16$ & & & \\
\hline $\mathrm{T}$ & 5.88 & 6.09 & 5.28 & 5.45 & $5.67 \pm 0.14$ & & & \\
\hline $\mathrm{PM} \pm \mathrm{SE}$ & $5.90 \pm 0.20$ & $5.95 \pm 0.10$ & $5.52 \pm 0.12$ & $5.52 \pm 0.17$ & & & & \\
\hline \multicolumn{9}{|c|}{ Cholesterol (mg/dL) } \\
\hline $\mathrm{NC}$ & 67.60 & 59.53 & 67.80 & 67.70 & $65.65 \pm 3.10$ & 0.093 & 0.010 & 0.923 \\
\hline $\mathrm{C}$ & 68.45 & 48.66 & 59.20 & 58.00 & $58.58 \pm 2.21$ & & & \\
\hline $\mathrm{T}$ & 65.30 & 52.68 & 61.74 & 59.86 & $59.89 \pm 2.02$ & & & \\
\hline $\mathrm{PM} \pm \mathrm{SE}$ & $67.12^{b} \pm 3.62$ & $53.62^{\mathrm{a}} \pm 2.52$ & $62.91^{\mathrm{b}} \pm 2.66$ & $61.85^{\mathrm{b}} \pm 1.89$ & & & & \\
\hline \multicolumn{9}{|c|}{ Creatinine (mg/dL) } \\
\hline $\mathrm{NC}$ & 0.87 & 0.85 & 1.05 & 0.98 & $0.94 \pm 0.07$ & 0.347 & 0.068 & 0.894 \\
\hline $\mathrm{C}$ & 0.86 & 0.91 & 1.26 & 1.40 & $1.11 \pm 0.09$ & & & \\
\hline $\mathrm{T}$ & 0.85 & 0.93 & 1.07 & 1.09 & $0.98 \pm 0.09$ & & & \\
\hline $\mathrm{PM} \pm \mathrm{SE}$ & $0.86^{\mathrm{a}} \pm 0.08$ & $0.90^{\mathrm{ab}} \pm 0.10$ & $1.13^{\mathrm{ab}} \pm 0.09$ & $1.16^{\mathrm{b}} \pm 0.10$ & & & & \\
\hline
\end{tabular}

${ }^{\mathrm{ab}}$ Means with different superscripts within a row differ significantly. $* \mathrm{NC}=$ Negative control; $\mathrm{C}=\mathrm{Control} ; \mathrm{T}=\mathrm{Treatment} ; \mathrm{d}=\mathrm{Days}$.

$\mathrm{PM}=$ Pooled mean, GM = Group mean.

al., 2008; Dubey et al., 2012). The LMM was found to be good source of $\mathrm{Ca}$. The condensed tannins content of LMM was 10.44 per cent.

\section{Intake and digestibility of nutrients}

The present results are in concurrence with the findings of Scharenberg et al. (2008), who reported non-significant difference in total intake of DM, OM and ADF in $H$. contortus infected lambs fed on diet with and without tanniferous sainfoin. The intake of DM by sheep was within the normal range (Kearl, 1982) and this clearly indicates that all the experimental diets were palatable. Fox et al. (1989) reported that DM digestibility was not affected due to Ostertagia infection in calves. Contrary to other reports, Rowe et al. (1988) reported a reduction in apparent digestibility of $\mathrm{OM}$ across the whole digestive tract, but particularly in the abomasum of $H$. contortus infected sheep. However, the extent of reduction of course depends upon the worm burden and the reduction is sometime small and often transitory in its nature. In the present study, decreased digestibility of $\mathrm{CP}$ and $\mathrm{EE}$ in $H$. contortus infected lambs supports earlier reports in $H$. contortus infected kids (Pathak and Tiwari, 2012a) and O. ostertagi infected calves (Entrocasso et al., 1986). This might be attributed to interference in absorption across the intestinal membrane, which coupled with the loss of protein coming from the disrupted mucosa passing through faeces.

\section{Nutritive value, plane of nutrition and their balances}

The absence of any detectable adverse effect on the health of experimental animals suggests that sheep were on balanced diets with no apparent deleterious consequences. The present results suggest that plane of nutrition was not affected adversely with CT supplementation are consistent with the earlier reports (Terrill et al., 1992; Dey et al., 2008). Nutrient density (\%) of composite diets in term of DCP and TDN also did not differ significantly among the groups. Many balance studies have demonstrated that reduced $\mathrm{N}$ retention is often a characteristic feature of GIN infection. This very often results from increased urinary $\mathrm{N}$ loss, implying a reduction in the efficiency of utilization of absorbed amino acids. In the present study $H$. contortus 
Table 6. Effect of condensed tannins on various serum enzymes activity in $H$. contortus infected sheep

\begin{tabular}{|c|c|c|c|c|c|c|c|c|}
\hline \multirow{2}{*}{ Attributes } & \multicolumn{4}{|c|}{ Periods* } & \multirow{2}{*}{$\mathrm{GM} \pm \mathrm{SE}$} & \multicolumn{3}{|c|}{$\mathrm{p}$ values } \\
\hline & $0 \mathrm{~d}$ & $30 \mathrm{~d}$ & $60 \mathrm{~d}$ & $90 \mathrm{~d}$ & & $\mathrm{G}$ & $P$ & $\mathrm{G} \times \mathrm{P}$ \\
\hline \multicolumn{9}{|l|}{$\overline{\mathrm{ALT}}$ (IU/L) } \\
\hline $\mathrm{NC}$ & 22.18 & 24.70 & 25.25 & 25.38 & $24.38 \pm 1.56$ & 0.527 & 0.302 & 0.988 \\
\hline $\mathrm{C}$ & 21.02 & 28.88 & 29.73 & 30.54 & $27.54 \pm 1.31$ & & & \\
\hline $\mathrm{T}$ & 22.53 & 25.74 & 26.27 & 26.76 & $25.32 \pm 2.70$ & & & \\
\hline $\mathrm{PM} \pm \mathrm{SE}$ & $21.91 \pm 3.02$ & $26.44 \pm 2.88$ & $27.08 \pm 1.21$ & $27.56 \pm 1.00$ & & & & \\
\hline \multicolumn{9}{|l|}{ AST (IU/L) } \\
\hline $\mathrm{NC}$ & 51.27 & 54.72 & 48.77 & 76.52 & $57.82 \pm 3.40$ & 0.810 & 0.000 & 0.986 \\
\hline $\mathrm{C}$ & 52.28 & 55.46 & 55.12 & 74.87 & $59.43 \pm 3.12$ & & & \\
\hline $\mathrm{T}$ & 53.36 & 51.87 & 50.07 & 72.90 & $57.05 \pm 3.07$ & & & \\
\hline $\mathrm{PM} \pm \mathrm{SE}$ & $52.30^{\mathrm{a}} \pm 2.25$ & $54.02^{\mathrm{a}} \pm 2.93$ & $51.32^{\mathrm{a}} \pm 2.07$ & $74.76^{b} \pm 3.95$ & & & & \\
\hline \multicolumn{9}{|l|}{$\operatorname{ALP}(\mathrm{IU} / \mathrm{L})$} \\
\hline $\mathrm{NC}$ & 144.85 & 146.18 & 151.35 & 156.43 & $149.70 \pm 5.99$ & 0.860 & 0.397 & 0.998 \\
\hline $\mathrm{C}$ & 146.52 & 143.88 & 162.06 & 169.29 & $155.44 \pm 9.07$ & & & \\
\hline $\mathrm{T}$ & 145.23 & 145.45 & 149.20 & 165.55 & $151.36 \pm 6.39$ & & & \\
\hline $\mathrm{PM} \pm \mathrm{SE}$ & $145.53 \pm 7.13$ & $145.17 \pm 8.47$ & $154.20 \pm 6.28$ & $163.76 \pm 10.62$ & & & & \\
\hline \multicolumn{9}{|l|}{ LDH (IU/L) } \\
\hline $\mathrm{NC}$ & 283.12 & 284.48 & 271.82 & 271.13 & $277.64 \pm 8.17$ & 0.787 & 0.633 & 1.000 \\
\hline $\mathrm{C}$ & 281.18 & 269.68 & 261.53 & 260.53 & $268.23 \pm 8.63$ & & & \\
\hline $\mathrm{T}$ & 283.40 & 282.37 & 266.85 & 265.13 & $274.44 \pm 10.70$ & & & \\
\hline $\mathrm{PM} \pm \mathrm{SE}$ & $282.57 \pm 11.17$ & $278.84 \pm 8.54$ & $266.73 \pm 11.59$ & $265.59 \pm 10.96$ & & & & \\
\hline
\end{tabular}

${ }^{\mathrm{ab}}$ Means with different superscripts within a row differ significantly. $* \mathrm{NC}=$ Negative control; $\mathrm{C}=\mathrm{Control} ; \mathrm{T}=\mathrm{Treatment} ; \mathrm{d}=\mathrm{Days}$.

$\mathrm{PM}=$ Pooled mean, $\mathrm{GM}=$ Group mean, $\mathrm{ALT}=$ Alanine aminotransferase .

$\mathrm{AST}=$ Aspartate aminotransferase, $\mathrm{ALP}=$ Alkaline phosphatase, $\mathrm{LDH}=$ Lactate dehydrogenase.

Table 7. Effect of condensed tannins on serum proteins and serum urea in $H$. contortus infected sheep

\begin{tabular}{|c|c|c|c|c|c|c|c|c|}
\hline \multirow{2}{*}{ Attributes } & \multicolumn{4}{|c|}{ Periods* } & \multirow{2}{*}{$\mathrm{GM} \pm \mathrm{SE}$} & \multicolumn{3}{|c|}{$\mathrm{p}$ values } \\
\hline & $0 \mathrm{~d}$ & $30 \mathrm{~d}$ & $60 d$ & $90 \mathrm{~d}$ & & $\mathrm{G}$ & $\mathrm{P}$ & $\mathrm{G} \times \mathrm{P}$ \\
\hline \multicolumn{9}{|c|}{ Total protein $(\mathrm{g} / \mathrm{dL})$} \\
\hline $\mathrm{NC}$ & 6.54 & 6.60 & 6.61 & 6.57 & $6.58^{\mathrm{b}} \pm 0.10$ & 0.000 & 0.099 & 0.058 \\
\hline $\mathrm{C}$ & 6.45 & 5.95 & 5.23 & 4.95 & $5.64^{\mathrm{a}} \pm 0.16$ & & & \\
\hline $\mathrm{T}$ & 6.39 & 6.82 & 6.28 & 6.59 & $6.52^{\mathrm{b}} \pm 0.18$ & & & \\
\hline $\mathrm{PM} \pm \mathrm{SE}$ & $6.46 \pm 0.08$ & $6.45 \pm 0.23$ & $6.04 \pm 0.19$ & $6.03 \pm 0.24$ & & & & \\
\hline \multicolumn{9}{|c|}{ Albumin (g/dL) } \\
\hline $\mathrm{NC}$ & 3.58 & 3.65 & 4.00 & 3.62 & $3.71^{\mathrm{b}} \pm 0.11$ & 0.000 & 0.000 & 0.000 \\
\hline $\mathrm{C}$ & 3.85 & 3.33 & 2.55 & 2.36 & $3.02^{\mathrm{a}} \pm 0.14$ & & & \\
\hline $\mathrm{T}$ & 3.82 & 3.38 & 2.93 & 2.88 & $3.25^{\mathrm{a}} \pm 0.10$ & & & \\
\hline $\mathrm{PM} \pm \mathrm{SE}$ & $3.75^{\mathrm{c}} \pm 0.10$ & $3.46^{\mathrm{b}} \pm 0.10$ & $3.16^{\mathrm{a}} \pm 0.17$ & $2.95^{\mathrm{a}} \pm 0.17$ & & & & \\
\hline \multicolumn{9}{|c|}{ Globulin (g/dL) } \\
\hline $\mathrm{NC}$ & 2.96 & 2.95 & 2.61 & 2.96 & $2.87^{\mathrm{ab}} \pm 0.11$ & 0.009 & 0.420 & 0.049 \\
\hline $\mathrm{C}$ & 2.60 & 2.62 & 2.69 & 2.59 & $2.62^{\mathrm{a}} \pm 0.10$ & & & \\
\hline $\mathrm{T}$ & 2.57 & 3.43 & 3.35 & 3.70 & $3.26^{\mathrm{b}} \pm 0.20$ & & & \\
\hline $\mathrm{PM} \pm \mathrm{SE}$ & $2.71 \pm 0.13$ & $3.00 \pm 0.21$ & $2.88 \pm 0.16$ & $3.08 \pm 0.20$ & & & & \\
\hline \multicolumn{9}{|c|}{ Urea (mg/dL) } \\
\hline $\mathrm{NC}$ & 42.75 & 40.54 & 40.73 & 40.62 & $41.16^{\mathrm{b}} \pm 0.80$ & 0.000 & 0.000 & 0.001 \\
\hline $\mathrm{C}$ & 42.92 & 41.38 & 42.51 & 42.13 & $42.24^{\mathrm{b}} \pm 0.56$ & & & \\
\hline $\mathrm{T}$ & 43.02 & 32.12 & 29.42 & 29.32 & $33.47^{\mathrm{a}} \pm 1.46$ & & & \\
\hline $\mathrm{PM} \pm \mathrm{SE}$ & $42.90^{b} \pm 0.75$ & $38.01^{\mathrm{a}} \pm 1.42$ & $37.56^{\mathrm{a}} \pm 1.68$ & $37.36^{\mathrm{a}} \pm 1.58$ & & & & \\
\hline
\end{tabular}

${ }^{\mathrm{abc}}$ Means with different superscripts within a row and column differ significantly.

*NC = Negative control; $\mathrm{C}=$ Control; $\mathrm{T}$ : Treatment; $\mathrm{d}=$ Days, PM = Pooled mean, GM: Group mean Jsul. 
Table 8. Comparative parameters of slaughtered sheep with $H$. contortus infection fed with and without CT supplemented diets

\begin{tabular}{|c|c|c|c|c|}
\hline \multirow{2}{*}{ Attributes } & \multicolumn{2}{|c|}{ Groups* } & \multirow{2}{*}{ SEM } & \multirow{2}{*}{$\mathrm{p}$ value } \\
\hline & $\mathrm{C}$ & $\mathrm{T}$ & & \\
\hline Body weight (kg) & 27.73 & 29.33 & 3.29 & 0.838 \\
\hline Fresh weight of meat $(\mathrm{kg})$ & 10.55 & 11.97 & 1.40 & 0.666 \\
\hline Weight of GIT (kg) & 9.41 & 9.57 & 1.15 & 0.952 \\
\hline Dressing percentage $(\%)$ & $37.72^{\mathrm{a}}$ & $40.66^{\mathrm{b}}$ & 0.81 & 0.048 \\
\hline
\end{tabular}

${ }^{\mathrm{ab}}$ Means with different superscripts within a row differ significantly. ${ }^{*} \mathrm{C}=\mathrm{Control}, \mathrm{T}=$ Treatment, GIT $=$ Gastrointestinal tract.

caused extensive abomasal tissue damage, haemorrhage and protein losses and redirected protein synthesis away from growth. Further, the restoration of damaged abomasal tissues leading to reduced $\mathrm{N}$ balance and increased serum urea level in infected control group further indicates poor reutilization of absorbed N. However, Coop (1982) and Symons (1982) suggested that loss of endogenous $\mathrm{N}$ into the intestine was the major reason for reduced $\mathrm{N}$ balance in their experiments in GIN infected animals. Moreover, CTs have adverse impact on GIN and their larvae (Waghorn, 1996). Several hypotheses have been proposed that CT has a direct and indirect effect on GIN in ruminants. Feeding of quebracho as CT source in sheep infected with $T$. circumcincta (Niezen et al., 1998a) and in goat infected with $H$. contortus, T. circumcincta and T. colubriformis (Paolini et al., 2003a, b; 2005) caused a reduction in FEC, which was associated with significant decreases in worm fecundity. The consumption of tanniferous legume forage by sheep (Niezen et al., 1998b) and goat (Paolini et al., 2005) was not only associated with an improved resistance of the host but also an improved resilience.

Similar to the present study, increased $\mathrm{N}$ retention in sheep and goats given tanniferous feeds at moderate levels due to lowered $\mathrm{N}$ excretion through urine has been reported earlier by several workers (Scharenberg et al., 2007, 2008; Dey et al., 2008). Another feature of $\mathrm{N}$ utilization as evident by significantly higher $\mathrm{N}$ retention as percent absorbed $\mathrm{N}$ (an indicator of availability of amino acids- $\mathrm{N}$ at tissue level) in animals given CT protected diets was apparently due to better amino acid availability and apparent biological value of CT protected diets (McDonald et al., 1995). The CT supplementation protects the protein in rumen from microbial hydrolysis and deamination and increases the proportion of dietary amino acids available for post-ruminal absorption. Ca and $\mathrm{P}$ intakes, outgo and their retention did not differ significantly irrespective of groups. However, slightly higher Ca intake $(\mathrm{p}<0.069)$ and balance $(\mathrm{p}<0.088)$ were recorded in CT fed group. In the present study, improved $\mathrm{Ca}$ intake and balance recorded in $\mathrm{T}$ group could be attributed to high Ca level in LMM as evidenced by the positive $\mathrm{Ca}$ and $\mathrm{P}$ balance in all experimental animals fed on tree leaves based diets (Kantwa et al., 2006; Ganai et al., 2007, 2010).

\section{Blood-biochemical profile}

Haematological parameters: The levels of $\mathrm{Hb}$ and PCV were reduced $(\mathrm{p}<0.001)$ after 30 days of experimental period in the infected control group reflecting the development of the disease in this group. However, CT supplementation to infected sheep (group $\mathrm{T}$ ) prevented a marked decrease in both the parameters. This suggests that the general health of infected sheep given CT was satisfactory throughout the experiment $(90 \mathrm{~d})$. Moore et al. (2008) observed that the feeding Sericea lespedeza (SL) hay in GI nematode infected goats improved PCV value as compared to bermuda grass (BG) fed goats. The present study revealed a marked reduction in $\mathrm{Hb}$ and $\mathrm{PCV}$ in infected control (C) which confirmed the observations of early worker (Misra et al., 1996) who observed decreased values of $\mathrm{Hb}$ and $\mathrm{PCV}$ in lambs in relation to nematode infection. The reduced $\mathrm{Hb}$ and PCV values in infected group may be attributed to the bleeding of abomasum due to injuries caused by the parasites (Abdel, 1992; Ahmed and Ansari, 1989).

Table 9. Comparison of local lymph nodes and parasitological parameters of slaughtered sheep with $H$. contortus infection fed with and without CT supplemented diets

\begin{tabular}{lrrrr}
\hline \multirow{2}{*}{ Attributes } & \multicolumn{2}{c}{ Groups* } & \multirow{2}{*}{ SEM } & p value \\
\cline { 2 - 5 } & \multicolumn{1}{c}{$\mathrm{C}$} & 2.49 & 0.16 & 0.502 \\
Pooled weight of PSLN $(\mathrm{g})$ & 2.24 & $2.26^{\mathrm{b}}$ & 0.15 & 0.038 \\
Pooled weight of ALN $(\mathrm{g})$ & $1.68^{\mathrm{a}}$ & $2,583.33^{\mathrm{a}}$ & $3,050.53$ & 0.045 \\
FECs & $1,3791.67^{\mathrm{b}}$ & $4,52.67^{\mathrm{a}}$ & 291.58 & 0.021 \\
Worm count/recovery & $1,597.67^{\mathrm{b}}$ & $83.33^{\mathrm{a}}$ & 3.30 & 0.002 \\
Larval development $(\%)$ & $97.59^{\mathrm{b}}$ & &
\end{tabular}

\footnotetext{
${ }^{\mathrm{ab}}$ Means with different superscripts within a row differ significantly.

$* \mathrm{C}=$ Control, $\mathrm{T}=$ Treatment, $\mathrm{ALN}$ all = Abomasal lymph nodes, PSLN = Pre scapular lymph nodes.

FECs $=$ Faecal egg counts.
} 
The mean eosinophil counts (\%) were significantly $(\mathrm{p}<0.001)$ higher in CT supplemented group followed by infected control and negative control. The period and treatment interaction was also significant $(p<0.001)$. Eosinophilia in infected groups in the present study are in agreement with the findings of Dawkins et al. (1989) and Kyriazakis et al. (1996) for T. colubriformis infected sheep or Etter et al. (2000) and Patterson et al. (1996 a,b) for infected goats or Pathak and Tiwari (2013) for H. contortus infected kids, who concluded that an increase in blood eosinophils is associated with the immune responsiveness of the animals to GI nematodes. Present results are contradictory with the findings of Paolini et al. (2003a, b) who reported no significant difference in blood eosinophils between tannin fed and control group of $H$. contortus, $T$. colubriformis and T. circumcincta infected goats. However, the number of inflammatory cells (Mast cells and globule leucocytes) was usually higher in the fundic mucosae from goats receiving tannins than in the control group (Paolini et al., 2003a). Similar to present findings (Hoskin et al., 2000) also recorded higher mean eosinophil counts in young deer fed legume forages containing CT in lungworm and GI nematode infected young deer as compared to control. Mean eosinophil counts of CT supplemented sheep (T) in this study exceeded the upper limits of the normal range, probably associated with the development of host immunity (Buddle et al., 1992). Hence in the current study, the higher blood eosinophil counts and lower FECs in H. contortus infected sheep receiving CT supplemented diet suggesting better expression of resistance or immune response of sheep with CT supplementation.

Biochemical parameters: Mean serum glucose $(\mathrm{mg} / \mathrm{dL})$ levels in all experimental sheep were within the normal range suggested for sheep (Boyd, 1984). Present results are in agreement with the finding of Cenci et al. (2007) who reported no difference in blood glucose levels in GIN infected sheep with and without CT supplementation from A. mearnsii. Similar to the present study comparable serum $\mathrm{Ca}$ and $\mathrm{P}$ levels in GIN infected lambs fed on diet with and without CT from Acacia mearnsii (Cenci et al., 2007). The serum cholesterol concentration and creatinine levels did not differ significantly $(p>0.05)$ among three different groups and was within the normal physiological range for sheep (Boyd, 1984). Similar to the present results, other reports also indicate no effect of CT supplementation on serum creatinine in goats and wethers (Yanez-Ruiz and Molina-Alcaide, 2008). Present results clearly pointed out that neither moderate level of CT (1.53\% of DM through LMM nor $H$. contortus infection in sheep interferes with the normal process of kidney during 90 days experimental period.

Serum urea level is an indicator of protein degradation in the rumen. Significantly lower level of serum urea in $\mathrm{T}$ group may be attributed to the reduced rumen protein breakdown and increased essential amino acid (EAA) absorption (Waghorn et al., 1990). Similarly, lower serum urea concentration was reported in lambs given diets containing CT through $F$. infectoria leaves at $1.5 \%$ to $2 \%$ (Dey et al., 2008) and in kids fed leaves of Prosopis cineraria (Bhatta et al., 2002). Similar variation in blood urea level in GIN infected sheep with and without CT supplemented diets from A. mearnsii have been reported by Cenci et al. (2007).

The reduction in total protein and albumin in $\mathrm{C}$ group and elevated level of globulin in $\mathrm{T}$ group might be attributed to the diversion of amino- $\mathrm{N}$ and energy from muscle, bone and collagen fibre. This in turn increases the endogenous losses of protein through urinary and faecal excretion. Some of it might be required for the synthesis of specific proteins for tissue repair and for immunological reaction to infection (MacRae, 1993). Present results are in conformity with the findings of many workers (Van Houtert and Sykes, 1996; Pathak and Tiwari, 2012b), who reported that sheep and kids offered a higher plane of nutrition are better able to withstand the pathological effects of nematode infection. Ahmed et al. (1990) also reported a decrease in total protein, albumin and an increase in Y-globulin levels in $H$. contortus infected sheep. It is possible to link the decrease level of total protein and albumin in infected control group which occurs as a result of abomasal haemorrhage caused by $H$. contortus. It is attributable to the loss of serum proteins into the gut and the subsequent impaired synthesis of albumin (Sharma et al., 2001) as a result of acute phase response associated with elevated level of globulin (Murata et al., 2004). Globulin levels increase when there is low blood albumin to counter balance the osmotic pressure (Payne and Payne, 1987). So it is imperative to get high globulin levels in GIN infected animals due to inflammation (Diogenes et al., 2010; Rumosa et al., 2010). CT supplementation in the present study reduced the FEC, so the effect of parasites on blood albumin and elevated level of globulin was minimized. However, the elevated globulin level in CT fed group may be due to indirect effect of $\mathrm{CT}$ to synthesize more immunoglobulins against $H$. contortus.

The main effect of GIN was associated with high fractional rates of removal of albumin as a result of elevated enteric loss of protein at the site of infection. In the present study CT supplementation of diet improved bioavailability of nutrients, decreased FECs and total worm counts and enabled infected sheep to improve their capacity to mount an effective immunological response which enhanced the onset of parasite rejection (Kyriazakis et al., 1994). In the present study parasites caused extensive protein losses and redirected protein synthesis away from skeletal muscle and to the repair of gut tissues leading to reduced $\mathrm{N}$ balance 
while elevated level of urea in $\mathrm{C}$ group indicated the poor reutilization of absorbed $\mathrm{N}$. Mean values of serum enzymes (ALT, AST, ALP, and LDH) were within the normal physiological range suggested for sheep (Kaneko, 1997). This clearly indicates that neither CT supplementation nor $H$. contortus infection in sheep throughout the entire feeding period have any adverse effect on target organs like liver, kidney and heart.

\section{Carcass yield and abomasal worm burden}

Pooled weight of ALN in T group was higher $(\mathrm{p}<0.05)$ than in the $\mathrm{C}$ group. Similar to present findings Balic et al. (2000) also observed a two-fold increase in weight of ALN in wool sheep $5 \mathrm{~d}$ after $H$. contortus infection. The increased weight of ALN in present study suggests greater infiltration and/or proliferation of immune cells in the abomasal tissues and lymph nodes. Increased number of immune cells could lead to greater parasite damage, which could explain the association between greater lymph node weights and lower FEC (MacKinnon, 2007). Present results are corroborated with findings of Min and Hart (2003) who reported a $76 \%$ reduction in total adult worm burden in Tracer goats grazed on L. cuneata compared to control. They further reported that L. cuneata diet resulted in a $94 \%$ reduction in $H$. contortus adults, a $100 \%$ reduction in Teladorsagia spp., and $45 \%$ lower numbers of Trichostrongylus. Similar results were also reported by Shaik et al. (2006) and Moore et al. (2008) for goats fed Sericea lespedeza compared with Burmuda grass hay. Significantly higher hatching percentage/ developed larvae $\left(\mathrm{L}_{3}\right)$ was observed in $\mathrm{C}$ group as compared to $\mathrm{T}$ group (Table 9). Similar to present results Molan et al. (1999, 2002) showed that CT extracts can disrupt the life cycle of nematodes by inhibition of egg hatching and larval development. Reduced hatchability will ultimately lead to less pasture contamination and reduced infection of grazing sheep. Shaik et al. (2006) and Moore et al. (2008) also reported lower percentage of larval development in goats fed SL hay compared with BG hay. The results clearly established the therapeutic role of CT against $H$. contortus infection in terms of reduced FECs, total worm counts and larval development in sheep.

\section{CONCLUSION}

It may be concluded that supplementation of CT $(1.5 \%$ of $\mathrm{DM})$ in $H$. contortus infected sheep has a discernible positive impact on N-retention and haematological parameters ( $\mathrm{Hb}$ and $\mathrm{PCV}$ ) without any adverse effects on nutrient utilization and blood-biochemical constituents. Moreover, CT supplementation in infected sheep had an inhibitory effect on intensity of $H$. contortus infection. Therefore, the tanniferous LMM may be used as part of a sustainable strategy to control GI nematodes in sheep.

\section{ACKNOWLEDGEMENT}

The financial assistance provided to the first author in the form of a fellowship by the Director, Indian Veterinary Research Institute, Izatnagar, India are gratefully acknowledged.

\section{REFERENCES}

Abdel Ali, T. S. 1992. Haematological and biochemical studies on efficiency of synthetic drugs against gastrointestinal nematode parasites in sheep. Aus. Vet. J. Med. 42:197-203.

Ahmad, A., C. Anwar-ul-Hassan, A. Anwar-ul-Haq, and M. A. Majeed. 1990. Serum proteinogram of lambs experimentally induced Haemonchus contortus infection. Vet. Arh. 60:195200.

Ahmad, M. and J. A. Ansari. 1989. Effect of Haemonchosis on haematology and non specific phosphomonoesterase activities in sheep and goats. Helminthol. 26:295-302.

Anbarasu, C., Narayan Dutta, K. Sharma, and M. Rawat. 2004. Response of goats to partial replacement of dietary protein by a leaf meal mixture containing Leucaena leucocephala, Morus alba and Tectona grandis. Small Rum. Res. 51:47-56.

Anonymous. 1984. Manual of veterinary investigation. Vol. 2 reference book 390. Ministry of Agriculture, Fisheries and Food. Her Majestry- stationary office, London, pp. 161-187.

AOAC. 2000. Official methods of analysis, Association of Official Analytical Chemists, $16^{\text {th }}$ edn, Washington, DC, USA.

Athanasiadou, S., I. Kyriazakis, F. Jackson, and R. L. Coop. 2001. Direct anthelmintic effects of condensed tannins towards different gastrointestinal nematodes of sheep: in vitro and in vivo studies. Vet. Parasitol. 99:205-219.

Balic, A., V. M. Bowles, and E. N. Meeusen. 2000. Cellular profiles in the abomasal mucosa and lymph node during primary infection with Haemonchus contortus in sheep. Vet. Immunol. Immunopathol. 75: 109-120.

Barham, D. and P. Trinder. 1972. An improved color reagent for the determination of blood glucose by the oxidase system. Analyst 97:142-145.

Bhatta, R., A. K. Shinde, S. Vaithiyanathan, S. K. Sankhyan, and D. L. Verma. 2002. Effect of polyethylene glycol-6000 on nutrient intake, digestion and growth of kids browsing Prosopis cineraria. Anim. Feed Sci. Technol. 101:45-54.

Boyd, J. W. 1984. The interpretation of serum biochemistry test results in domestic animals. Vet. Clin. Pathol. 13:7-14.

Bretaudiere, J. P., A. Vassault, L. Amsellem, M. L. Pourci, H. Thiece-Phung, and M. Bailly. 1977. Criteria for establishing a standardized method for determining alkaline phosphatase activity in human serum. Clin. Chem. 23:2263-2274.

Buddle, B. M., G. Jowett, R. S. Green, P. G. Douch, and P. L. Risdon. 1992. Association of blood eosinophilia with the expression of resistance in Romney lambs to nematodes. Int. J. Parasitol. 22: 955-960.

Cenci, F. B., H. Louvandini, C. M. McManus, A. Dell'Porto, D. M. Costa, S. C. Araujo, A. P. Minho, and A. L. Abdalla. 2007. Effects of condensed tannin from Acacia mearnsii on sheep 
infected naturally with gastrointestinal helminthes. Vet. Parasitol. 144:132-137.

Chaichisemsari, M., B. Eshratkhah, N. Maherisis, M. Sadaghian, and S. Hassanpour. 2011. Evaluation of total protein, albumin, globulin and blood urea nitrogen concentrations in gastrointestinal nematodes infected sheep. Global Vet. 6:433437.

Chandrawathani, P., M. Adnan, and C. M. Zaini. 2000. Preliminary study on Neem (Azadirachta indica) as an alternative anthelmintic for sheep. Proc. 12th Vet. Assoc. Malaysia Scientific Cong., Malaysia, Kuanyan.

Coop, R. L. 1982. The impact of sub clinical parasitism in ruminants. In: Parasites-Their world and Ours (Ed. D. F. Mettrick and S. S. Desser) Amsterdam: Elsevier, pp. 439-450.

Dacie, Z. and S. Lewis. 1969. Practical Heamatology $4^{\text {th }}$ ed. J. and A., Churchill, London.

Dawkins, H. J. S., R. G. Windon, and G. K. Eagleson. 1989. Eosinophil responses in sheep selected for high and low responsiveness to Trichostrongylus colubriformis. Int. J. Parasitol.19:199-205.

Dey, A., N. Dutta, K. Sharma and A.K. Pattanaik. 2008. Effect of dietary inclusion of Ficus infectoria leaves as a protectant of proteins on the performance of lambs. Small Rumin. Res. 75: 105-114.

Diogenes, P. V. A., A. C. D. Suassuna, S. M. M. Ahid and B. SotoBlanco. 2010. Serum protein electrophoretic profile of goats infected with Haemonchus contortus. J. Anim. Vet. 9:16031606.

Doumas, B. T., W. A. Watson, and H. G. Biggs. 1971. Albumin standards and the measurements of serum albumin with bromocresol green. Clin. Chem. Acta. 31:87-96.

Dubey, M., N. Dutta, P. S. Banerjee, A. K. Pattanaik, K. Sharma, and M. Singh. 2012. Effect of condensed tannin supplementation through a tree leaves mixture on erythrocytic antioxidant status and gastrointestinal nematodes in kids. Anim. Nutr. Feed Technol. 12:91-102.

Entrocasso, C. M., J. J. Parkins, J. Armour, K. Bairden, and P. N. McWilliam. 1986. Metabolism and growth studies in housed calves given a morantel sustained release bolus and exposed to natural trichostrongyle infection. Res. Vet. Sci. 40:65-75.

Etter, E., H. Hoste, C. Chatier, I. Pors, C. Koch, C. Broqua, and H. Coutineau. 2000. The effect of two levels of dietary protein on resistance and resilience of dairy goats experimentally infected with Trichostrongylus colubriformis: comparison between high and low producers. Vet. Res. 31:247-258.

Fox, M. T., D. Gerelli, S. R. Pett, D. E. Jacob, M. Gill, and D. L. Gale. 1989. Ostertagia ostertagi infection in the calf: effects of a trickle challenge on appatite, digestibility, rate of passage of digesta and live weight gain. Res. Vet. Sci. 47:294-298.

Ganai, A. M., H. A. Ahmad, and S. Bilal. 2010. Nutritional evaluation of green mulberry (Morus multicaulis) leaves in sheep. Anim. Nutr. Feed Technol. 10: 133-138.

Ganai, A. M., H. A. Ahmad, J. I. A. Bhat, and S. Bilal. 2007. Nutritional evaluation of green popular (Populus deltoids) foliage in sheep. Appl. Biol. Res. 9:34-38.

Hallett, C. J. and J.G. H. Cook. 1971. Produced nicotinamide adenine dinucleotide- coupled reaction for emergency blood urea estimation. Clin. Chem. Acta. 35:33-37.

Hassanpour, S., B. Eshratkhah, M. Sadaghian, N. Maherisis, M.
Chaichisemsari, and M. kiyaninahand. 2011. Relationship between gastrointestinal parasite infection and faecal protein excretion in Moghani ewes. Int. J. Anim.Vet. Adv. 3:167-169.

Hoskin, S. O., P. R. Wilson, T. N. Barry, W. A. G. Charleston, and G. C. Waghorn. 2000. Effect of forage legumes containing condensed tannins on lungworm (Dictyocaulus sp.) and gastrointestinal parasitism in young red deer (Cervus elaphus). Res. Vet. Sci. 68:223-230.

IFOM. 2006. Proceedings of the first international conference of animal in organic production, Minnesota University, St. Paul, Minnesota, USA August, 23-25.

Kaneko, J. J. 1997. Clinical biochemistry of domestic animals. 5th edn, (Academic Press, New York, USA), pp. 885-905.

Kantwa, L. C., L. Gupta, B. P. Singh, and S. P. Tailor. 2006. Nutritional evaluation of mulberry leaves (Morus alba) in sheep and goat. Indian J. Small Rum. 12:202-205.

Kearl, L. C. 1982. Nutrient requirements of ruminants in developing countries. International Feed Stuffs Institute, Utah Agricultural Experimental Station, (Utah State University, Logan, Utah- 84322, USA), pp. 45-81.

Kyriazakis, I., D. H. Anderson, R. L. Coop, and F. Jackson. 1996. The pathophysiology and development of immunity during long-term subclinical infection with Trichostrongylus colubriformis of sheep receiving different nutritional treatments. Vet. Parasitol. 65:41-54.

Kyriazakis, I., J. D. Oldham, R. L. Coop, and F. Jackson. 1994. The effect of subclinical intestinal nematode infection on the diet selection of growing sheep. Br. J. Nutr. 72:665-677.

Leng, R. A. 1990. Factors affecting the utilization of poor quality forages by ruminants particularly under tropical condition. Nutr. Res. Rev. 3:277-303.

MacKinnon, K. M. 2007. Differential gene expression and immune regulatory mechanism in parasitic-resisitant hair and susceptible wool sheep infected with the parasitic nematode, Haemonchus contortus. Ph D. Thesis. Faculty of Virginia Polytechnic Institute and State University, Blackberg, Virginia.

MacRae, J. C. 1993. Metabolic consequence of intestinal parasitism. Proc. Nutr. Soc. 52:121-130.

Makkar, H. P. S. 2000. Quantification of Tannins in Tree Foliage A Laboratory Manual (FAO/IAEA Working Document, Vienna, Austria).

McDonald, P., R. A. Edwards, J. F. D. Greenhalgh, and C. A. Morgan. 1995. In: Animal Nutrition $5^{\text {th }}$ edn, (Longman Scientific and Technical Inc., New York).

Min, B. R. and S. P. Hart. 2003. Tannins for suppression of internal parasites. J. Anim. Sci. 81:E102-E109.

Misra, S. C., D. N. Panda, and S. Psida. 1996. Haematological and histological alterations of immature Paramphistomiasis in lambs. Indian Vet. J. 73:1274-1276.

Molan, A. L., G. C. Waghorn, and W. C. McNabb. 1999. Condensed tannins and gastrointestinal nematodes in sheep. Proc. NZ. Grassl. Assoc. 61:57-61.

Molan, A. L., G. C. Waghorn, and W. C. McNabb. 2002. Effect of condensed tannins on egg hatching and larval development of Trichostrongylus colubriformis in vitro. Vet. Rec. 150:65-69.

Moore, D. A., T. H. Terrill, B. Kouakou, S. A. Shaik, J. A. Mosjidis, J. E. Miller, M. Vanguru, G. Kannan, and J. M. Burke. 2008. The effects of feeding sericea lespedeza hay on growth rate of goats naturally infected with gastrointestinal 
nematodes. J. Anim. Sci. 86:2328-2337.

Murata, H., N. Shimada, and M. Yoshika. 2004. Current research on acute phase proteins in veterinary diagnosis: An overview. Vet. J. 168:28-40.

Newman, D. J. and C. P. Price. 1999. Renal function and nitrogen metabolites. In: Tietz Textbook of Clinical Chemistry (Ed. C. A. Burtis and E. R. Ashwood), $3^{\text {rd }}$ edn, Philadelphia: W B Saunders Company, pp. 1204.

Niezen, J. H., G. C. Waghorn, and W. A. G. Charleston. 1998a. Establishment and fecundity of Ostertagia circumcincta and Trichostrongylus colubriformis in lambs fed Lotus ( $L$. pedunculatus) or perennial ryegrass (Lolium perenne). Vet. Parasitol. 78:13-21.

Niezen, J. H., H. A. Robertson, G. C. Waghorn, and W. A. G. Charleston. 1998b. Production, faecal egg counts and worm burdens of ewe lambs which grazed six contrasting forages. Vet. Parasitol. 80:15-27.

Pandey, V. S., W. Pralomkram, S. Kochapakdee, and S. Saithanoo. 2001. Benzimidazol resistance in Haemonchus contortus from goat in Thailand. PSU Goat Res. Pub. pp. 337.

Paolini, V., A. Frayssines, F. D. L. Farge, P. Dorchies, and H. Hoste. 2003a. Effects of condensed tannins on established populations and on incoming larvae of Trichostrongylus colubriformis and Teladorsagia circumcincta in goats. Vet. Res. 34:331-339.

Paolini, V., F. De La Farge, F. Prevot, P. Dorchies, and H. Hoste. 2005. Effects of the repeated distribution of sainfoin hay on the resistance and the resilience of goats naturally infected with gastrointestinal nematodes. Vet. Parasitol. 127:277-283.

Paolini, V., J. P. Bergeaud, C. Grisez, F. Prevot, P. Dorchies, and H. Hoste. 2003b. Effects of condensed tannins on goats experimentally infected with Haemonchus contortus. Vet. Parasitol. 113:253-261.

Parkins, J. J. and P. Holmes. 1989. Effects of gastrointestinal helminth parasites on ruminant nutrition. Nutr. Res. Rev. 2: 227-246.

Pathak, A. K. 2011. Nutritional status and performance of ruminants as influenced by gastrointestinal nematodes: An overview. NE Vet. 11:20-26.

Pathak, A. K. and S. P. Tiwari. 2012a. Influence of Haemonchus contortus infection on nutrient intake and its utilization in kids fed different levels of nutrition. Indian J. Anim. Nutr. 29:52-57.

Pathak, A. K. and S. P. Tiwari. 2012b. Influence of Haemonchus contortus on biochemical profile in kids fed on different diets. Vet. Pract. 13:63-65.

Pathak, A. K. and S. P. Tiwari. 2013. Effect of high plane of nutrition on the performance of Haemonchus contortus infected kids. Vet. World. 6:22-26.

Pathak, A. K. and S. Pal. 2008. Seasonal prevalence of gastrointestinal parasites in goats from durg district of Chhattisgarh. Vet. World 1:136-137.

Patra, A. K., K. Sharma, N. Dutta, and A. K. Pattanaik. 2006. Effect of partial replacement of dietary protein by a leaf meal mixture on nutrient utilization by goats in pre and late gestation. Small Rumin. Res. 63:66-74.

Patterson, D. M., F. Jackson, J. F. Huntley, L. M. Stevenson, D. G. Jones, E. Jackson, and J. F. Russel. 1996a. Studies on caprine responsiveness to Nematodiasis: segregation of male goats into responders and non-responders. Int. J. Parasitol. 26:187-194.
Patterson, D. M., F. Jackson, J. F. Huntley, L. M. Stevenson, D. G. Jones, E. Jackson, and J. F. Russel. 1996a. The response of breeding doses to Nematodiasis: segregation into responders and non-responders. Int. J. Parasitol. 26:1295-1303.

Payne, J. M. and S. Payne. 1987. The metabolic profile test. Oxford University Press, Oxford (1987) p. 179.

Richmond, W. 1973. Cholesterol: oxygen oxidoreductase enzyme catalyzes oxidation of cholesterol to 4-cholesten-3-one with production of hydrogen peroxides and $\mathrm{H}_{2} \mathrm{O}_{2}$ react with PAP reagent. Clin. Chem. 19:1350.

Rowe, J. B., J. V. Nolan, G. de Chaneet, E. Teleni, and P. H. Holmes. 1988. The effect of haemonchosis and blood loss into the abomasum on digestion in sheep. Br. J. Nutr. 59:125-139.

Rumosa, Gwaze, F., M. Chimonyo, and K. Dzama. 2010. Relationship between nutritionally-related blood metabolites and gastrointestinal parasites in Nguni goats of South Africa. Asian-Aust. J. Anim. Sci. 23:1190-1197.

Sastary, G. A. 1989. Veterinary clinical pathology. CBS Publishers and Distributors Pvt. Ltd., Delhi, India

Scalbert, A. 1991. Antimicrobial properties of tannin. Phytochemistry 30:3875-3883.

Scharenberg, A., F. Heckendorn, Y. Arrigo, H. Hertzberg, A. Gutzwiller, H. D. Hess, M. Kreuzer, and F. Dohme. 2008. Nitrogen and mineral balance of lambs artificially infected with Haemonchus contortus and fed tanniferous sainfoin (Onobrychis vicifolia). J. Anim. Sci. 86:1879-1890.

Scharenberg, A., Y. Arrigo, A. Gutzwiller, U. Wyss, H. D. Hess, M. Kreuzer, and F. Dohme. 2007. Effect of feeding dehydrated and ensiled tanniferous sainfoin (Onobrychis vicifolia) on nitrogen and mineral digestion and metabolism of lambs. Arch. Anim. Nutr. 611:390-405.

Shaik, S. A., T. H. Terrill, J. E. Miller, B. Kouakou, G. Kannan, R. M. Kaplan, J. M. Burke, and J. A. Mosjidis. 2006. Sericea lespedeza hay as natural deworming agent against gastrointestinal nematode infection in goats. Vet. Parasitol. 139:150-157.

Sharma, D. K., P. P. S. Chauhan, and R. D. Agarwal. 2001. Changes in the levels of serum enzymes and total protein during experimental haemonchosis in Barbari goats. Small Rumin. Res. 42:119-123.

Snedecor, G. W. and W. G. Cochran. 1994. Statistical methods, $8^{\text {th }}$ edn. East West Press Pvt. Ltd., New Delhi.

Symons, L. E. A. 1982. Protein synthesis in the whole body, liver and skeletal muscle. In Parasites-Their world and Ours, pp. 233-241 (Ed. D. F. Mettrick and S. S. Desser) Amsterdam, Elsevier.

Talpatra, S. K., S. C. Ray, and K. C. Sen. 1940. Estimation of phosphorus, chlorine, calcium, magnesium, sodium and potassium in foodstuffs. Indian J. Vet. Sci. Anim. Husb. 10: 243-258.

Terrill, T. H., G. B. Douglas, A. G. Foote, R. W. Purchas, G. F. Wilson, and T. N. Barry. 1992. Effect of condensed tannins upon body growth, wool growth and rumen metabolism in sheep grazing sulla (Hedysarum cornarium) and perennial pasture. J. Agric. Sci. Camb. 119:265-273.

Thefeld, W., H. Hoffmeister, E. W. Busch, P. U. Koller, and J. Vollmar. 1974. Referenzwerte fur die Bestimmungen der Transaminasen GOT und GPT sowie der alkalischen phosphatase im serum mit optimierten standardmethoden. 
Dtsch. Med. Wochenschr. 99:343-351.

Urquhart, G. M., J. Armour, J. L. Duncan, A. M. Dunn, and F. W. Jennings. 1996. Veterinary parasitology, 2nd edn. (Blackwell Science Ltd) pp. 276-278.

Van Houtert, M. F. J. and A. R. Sykes 1996. Implications of nutrition for the ability of ruminants to withstand gastrointestinal nematode infections. Int. J. Parasitol. 26:11511167.

Van Soest, P. J., J. B. Robertson, and B. A. Lewis. 1991. Methods for dietary fibre, neutral detergent fibre and non-starch polysaccharides in relation to animal nutrition. J. Dairy Sci. 74: 3583-3597.

Vatzidis, H. 1977. An improved biuret reagent. Clin. Chem. 23: 908.
Waghorn, G. C. 1996. Condensed tannins and nutritive absorption from small intestine. Proc. Can. Soc. Anim. Sci., Lethbridge, Alberta, Canada. L. M. Rode, edn.

Waghorn, G. C., I. D. Shelton, and B. R. Sinclair. 1990. Distribution of elements between solid and supernatant fractions of digesta in sheep given six diets. NZ. J. Agric. Res. 33:259-269.

Wroblewski, V. F. and L. S. Duean. 1955. Bestimmung der Aktivital der lactate dehydragenase. Proc. Soc. Exp. Biol. 90: 210-214.

Yanez-Ruiz, D. R. and E. Molina-Alcaide. 2008. A comparative study of nutrients utilization, alkaline phosphatase activity and creatinine concentration in the serum of sheep and goats fed diets based on olive leaves. J. Anim. Physiol. Anim. Nutr. 92: 141-148. 